\title{
Vidas. Création d'un dictionnaire biographique des acteurs de la renaissance d'oc en ligne : nécessité et
} contraintes

Vidas: the need for and the developmental constraints of a biographical dictionary of actors involved in the revitalisation of the langue d'oc online

\section{Yan Lespoux}

\section{OpenEdition Journals}

Édition électronique

URL : http://journals.openedition.org//bl/447

DOI : $10.4000 / / b l .447$

ISSN : 2727-9383

Éditeur

Université de Bretagne Occidentale - UBO

\section{Édition imprimée}

Date de publication : 1 mars 2016

Pagination : 191-198

ISBN : 979-10-92331-24-0

ISSN : $1270-2412$

\section{Référence électronique}

Yan Lespoux, «Vidas. Création d'un dictionnaire biographique des acteurs de la renaissance d'oc en ligne : nécessité et contraintes », La Bretagne Linguistique [En ligne], 20 | 2016, mis en ligne le 01 mai 2020, consulté le 01 octobre 2020. URL : http://journals.openedition.org//bl/447 ; DOI : https://doi.org/ $10.4000 / \mathrm{lbl} .447$

\section{(c) (†)}

La Bretagne Linguistique est mise à disposition selon les termes de la Licence Creative Commons Attribution 4.0 International. 


\title{
Vidas. Création d'un dictionnaire biographique des acteurs de la renaissance d'oc en ligne : nécessité et contraintes
}

\author{
Yan LESPOUX
}

\section{Genèse du projet}

La renaissance d'oc, de la première moitié $d u x x^{e}$ siècle à nos jours a été le fait d'une multitude de personnages, plus ou moins impliqués dans ce mouvement et, surtout, plus ou moins connus en dehors de cercles militants parfois très circonscrits. Historiens et chercheurs en domaine occitan se heurtent régulièrement à l'absence ou à la difficulté d'accéder à des sources biographiques concernant ces personnalités.

Pour avoir fait régulièrement l'expérience de ces manques, mais pour savoir aussi que les sources sont toutefois nombreuses, les chercheurs de l'équipe $\mathrm{ReDoc}$ (Recherche en domaine occitan), composante de l'équipe LLACS (Langues, littératures, arts et cultures du sud) de l'Université Montpellier 3 ont émis l'idée de produire un dictionnaire biographique des acteurs de la renaissance d'oc. Car s'il existe, certes, un certain nombre de dictionnaires bibliographiques, ceux-ci, par la force des choses, ne s'intéressent qu'aux personnages ayant produit des livres et brochures en occitan et s'attardent généralement peu sur les éléments biographiques. Or, si la littérature est le plus visible des engagements dans cette renaissance, il n'est pas le seul. Et nombreux sont les personnages qui ont pris leur part dans le mouvement en ne publiant que dans des périodiques, revues, almanachs ou journaux locaux, en animant des cercles locaux, en s'engageant en politique plus qu'en littérature, ou en écrivant sans utiliser l'occitan. Autant d'individus, donc, qui tendent à passer sous les radars des dictionnaires bibliographiques et que l'on croise au long de l'histoire de la renaissance d'oc sans pouvoir toujours les identifier avec précision. Par ailleurs, l'idée d'un dictionnaire biographique centré sur les engagements est propre à dynamiser les travaux historiographiques qui sont aujourd'hui le parent pauvre des études occitanes.

Un modèle existe en la matière. Il s'agit du monumental Dictionnaire biographique du mouvement ouvrier lancé il y a un demi-siècle 
par Jean Maitron ${ }^{1}$ et encore inachevé. Cet aspect de work in progress du Maitron que revêtirait obligatoirement un dictionnaire biographique du mouvement d'oc nous a poussé à exclure assez rapidement l'idée d'une publication papier. Un choix conforté par le fait que le CIRDÒC (Centre inter régional de documentation occitane), pôle associé à la Bibliothèque nationale de France, s'est lancé depuis 2006 dans un important chantier de constitution d'instruments de recherches collectifs. Ainsi s'élaborent des outils d'information et de documentation de référence et se fédèrent, au sein de bases de connaissances, les données et savoirs produits par les bibliothèques, archives, centres de documentation spécialisés, musées, aussi bien que les universités, composantes de recherche et chercheurs individuels dans le cadre du mouvement des archives ouvertes scientifiques. Disposant de ressources et de compétences informatiques que les chercheurs de I'Université de Montpellier n'avaient pas, le CIRDÒC apparaissait donc comme un partenaire essentiel à la mise en place de ce projet et son directeur, Benjamin Assié a accepté avec enthousiasme d'associer l'établissement à ce projet.

1. Jean MAITRON, Dictionnaire biographique du mouvement ouvrier français, Paris, Éditions ouvrières, 1964. Voir : http:// biosoc.univ-paris1.fr/ et http://maitronen-ligne.univ-paris1.fr/ (sites consultés le 20 novembre 2015).
Ainsi, de la synergie entre l'équipe ReDoc et le CIRDÒC, grand centre de documentation constituant une source de données et de documents incomparables complétée par le programme Occitanica $^{2}$ qui informatise et fédère les données et ressources de plus de 70 établissements partenaires, est né, il y a un peu plus de deux ans, le projet Vidas ${ }^{3}$.

\section{La mise en place}

La première question qui se pose dans le cadre de ce genre de projet est bien entendu celle des sources.

Les nécrologies des auteurs ou des militants les plus importants fournissent un certain nombre de renseignements, pas forcément suffisants, produits dans des circonstances où la priorité est de rendre hommage. Rares sont ceux qui ont fait l'objet de travaux de nature biographique; moins rares peut-être ceux qui ont laissé des témoignages autobiographiques, dont la fiabilité est, par nature, discutable. Certains ont laissé des archives (plus ou moins) aisément consultables, qui permettent d'en savoir plus sur eux-mêmes et sur leurs correspondants ou partenaires. Les fonds d'archives

2. Métaportail et bibliothèque numérique de la documentation occitane. Accessible à l'adresse : http://www.occitanica. eu/index.php/fr/ (site consulté le 20 novembre 2015).

3. Accessible à l'adresse : http://vidas. occitanica.eu/ (site consulté le 20 novembre 2015). 
privées des personnalités occitanistes déposés au CIRDÒC, dans les bibliothèques, les archives départementales, ne sont pas non plus toujours fiables. II est notamment rare que le donateur rende public sans tri la totalité de ses engagements politiques, idéologiques, d'autant plus lorsqu'ils ont évolué au cours de sa vie.

En somme, les sources existent. Comme existent les chercheurs susceptibles de les exploiter, de les confronter, de les interroger : tous ont produit sur tel ou tel aspect, telle ou telle région particulière, telle ou telle période de I'histoire qu'il s'agit de raconter. Chacun d'eux a amassé au cours de ses recherches une documentation qui lui permet de cerner la personnalité des individus qu'il a croisés. Le spécialiste de littérature connaît les auteurs dont il traite. L'historien a identifié les militants importants et le réseau dans lequel ils s'insèrent. II appartient à présent de réunir toutes ces compétences et tous ces savoirs dans une entreprise collective.

II s'agit donc d'une entreprise de longue haleine dans laquelle il serait vain d'espérer déboucher dans un délai raisonnable sur un ouvrage papier prétendant à l'exhaustivité. De là l'idée d'une banque de données évolutive, complétée progressivement au rythme de la production des fiches individuelles et rendant par ailleurs possible les ajouts d'informations dans les fiches déjà exis- tantes au fur et à mesure des avancées de la recherche.

Cette forme générale adoptée, il convient alors de mettre en place un réseau de collaborateurs sur le modèle du Maitron, c'est-àdire par zone géographique et/ou par secteur d'activité (enseignement, presse, art...).

Pour ce faire, un appel à collaboration a été lancé accompagné de modèles de fiches élaborés par les initiateurs du projet. Ces fiches doivent être aussi complètes que possibles sans pour autant être trop longues (il ne s'agit pas d'écrire des biographies de dizaines ou centaines de pages mais bien des fiches biographiques); ainsi, en fonction de l'importance du personnage traité et des sources disponibles, la fiche peut être plus ou moins fournie, de quelques centaines de signes jusqu'à 20000, selon les cas. Surtout, par souci de cohérence, les fiches doivent toute suivre la même structure :

- Le nom du personnage et ses dates et lieu de naissance et de mort ;

- Ses pseudonymes connus;

- Un résumé de la fiche ;

- Une partie consacrée aux éléments biographiques généraux (milieu d'origine, métier...) ;

- Une partie spécifiquement consacrée aux engagements dans la renaissance d'oc ;

- Une bibliographie du personnage si elle existe ;

- Les sources qui ont servi à l'élaboration de la fiche ; 
- Une photographie ou un portrait lorsqu'ils sont disponibles.

Par ailleurs, un menu, sur le côté de la fiche, indique les associations (Félibrige, Institut d'Études Occitanes, cercles locaux...) dont le personnage a été membre, ses engagements politiques ou sociaux (parti, syndicat, franc-maçonnerie...), son ou ses métiers ainsi que les lieux géographiques qu'il a fréquentés et la ou les périodes historiques dans laquelle ou lesquelles il s'insère. $\mathrm{Ce}$ menu doit permettre d'effectuer des recoupements, de faire apparaître les éventuels réseaux de personnes ou même, à terme, de faire des études statistiques sur les acteurs de la renaissance d'oc (cf. illustration 1).

Il s'agit là du cœur du projet Vidas qui doit permettre à la fois d'éclairer sur des personnages particuliers et de mettre en lumière les réseaux dans lesquels ils s'insèrent. Le cas de Pierre-Louis Berthaud, première fiche réalisée (cf. illustration 2) dans le cadre de ce projet est l'exemple type de ce à quoi peut et doit servir ce dictionnaire biographique.

Pierre-Louis Berthaud n'est pas écrivain et les seuls ouvrages dont il est l'auteur, essentiellement des bibliographies, ne dévoilent que très peu son implication dans la renaissance d'oc. Personnage discret qui est longtemps demeuré dans l'ombre, il n'en est pas moins l'un des acteurs les plus importants de la revendication d'oc dans les années 1940 et 1950. Lié à la fois aux deux grandes associations antagonistes de l'après-guerre, le Félibrige et I'Institut d'études occitanes, il est aussi journaliste, syndic de la presse parlementaire ce qui fait de lui un des acteurs essentiels, avec le Breton Léon Toulemont du combat pour le vote de la loi Deixonne sur l'enseignement des langues et dialectes locaux député gaulliste à l'Assemblée de I'Union Française et secrétaire de l'amicale des anciens de Dachau. Pour avoir par ailleurs organisé à la fin des années 1930 l'accueil des intellectuels catalans réfugiés en France, il dispose de nombreux contacts catalans. Outre qu'elle va pouvoir offrir au chercheur ou au curieux une biographie riche basée sur des sources de première main (la correspondance même de Berthaud), sa fiche commence à faire apparaître les réseaux qui se tissent autour de Pierre-Louis Berthaud et des groupements qu'ils fréquentent. Mise en relation avec d'autres fiches au fur et à mesure qu'elles seront rédigées et publiées sur le site et complétée au rythme des compléments que pourront apporter de nouveaux fonds documentaires, elle permettra de mieux mettre en lumière ces réseaux et de mieux relier entre eux les acteurs de cette période tout comme ceux des périodes précédentes et suivantes avec lesquels Berthaud a pu tisser des liens dans sa jeunesse ou au contraire au crépuscule de sa vie. 


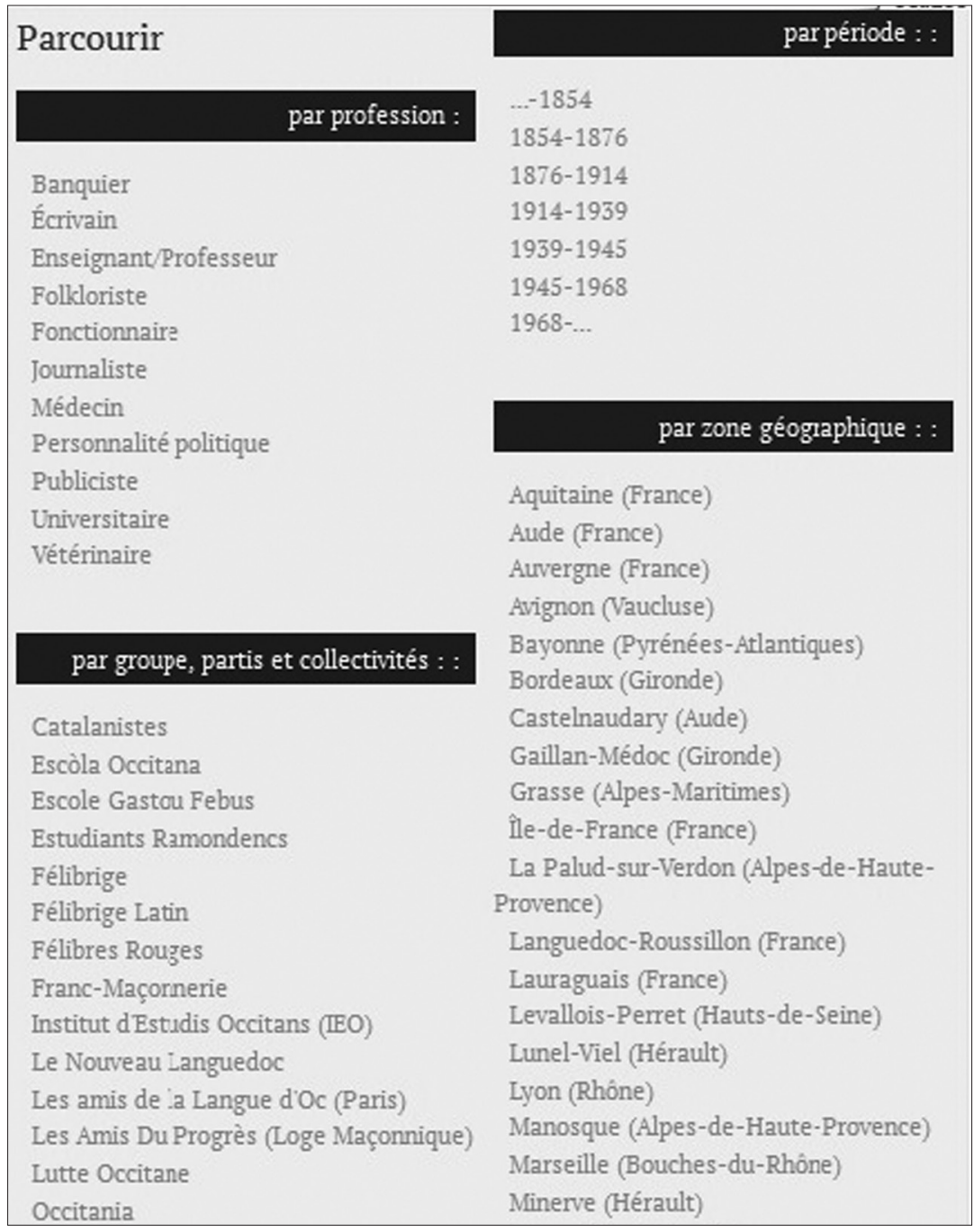

Illustration 1.

Il en va de même d'Ismaël Girard, autre éminence grise de l'occitanisme, médecin, touchant peu à l'écriture si ce n'est par le biais de quelques articles dans des revues occitanes ou catalanes, mais qui a pourtant lancé, dirigé et financé nombre de revues et mouvements essentiels de la re- naissance d'oc mais qui reste cependant un quasi inconnu pour une grande partie des étudiants et chercheurs. Là encore, sa fiche mise en ligne permet à la fois de faire connaissance avec le personnage mais aussi de mieux comprendre l'occitanisme durant les périodes concernées. 


\begin{tabular}{|c|c|}
\hline \multirow[t]{2}{*}{ Pierre-Louis BERTHAUD (1899-1956) } & Dictionnaire \\
\hline & biographique des acteurs \\
\hline \multirow{3}{*}{$\begin{array}{l}\text { Sommaire } \\
\text { Éléments biographiques } \\
\text { Engagements dans la renaissance d'oc }\end{array}$} & de la Renaissance \\
\hline & occitane \\
\hline & avancée \\
\hline \multirow{3}{*}{$\begin{array}{l}\text { Pierre-Louis Berthaud (Bordeaux,Gironde, } 24 \text { août 1899, Séry-Magneval, Oise, } 8 \text { août 1956), joumaliste, homme politique, } \\
\text { majoral du Félibrige (Cigale du Tam), membre de l'Institut d'Études Occitanes (IEO), franc-maçon, cofondateur de la revue } \\
\text { Occitania (1956). } \\
\text { Éléments biographiques }\end{array}$} & uteur(s) de l'article \\
\hline & Lespoux, Yan \\
\hline & Sujets associés \\
\hline \multirow{4}{*}{$\begin{array}{l}\text { Fils d'instituteurs, i étudie à Bordeaux où il obtient une licence de Droit et Lettres. Mobilisé en tant que traducteur auprès des } \\
\text { forces américaines en 1918, il est alors membre de la SFro. Son ascension dans la fédération socialiste de la Gironde est } \\
\text { d'ailleurs rapide puisqu'il assiste au congrès de Tours de } 1920 \text { en tant que délégué (bien que son mandat semble ne pas avoir } \\
\text { été validé). Mais il se trouve éloigné de la vie militante pendant plusieurs années suite à un grave accident d'automobile auquel } \\
\text { vient s'ajouter une tuberculose. }\end{array}$} & $\begin{array}{l}\text { Parcourir par métiers : } \\
\text { Journaliste }\end{array}$ \\
\hline & Personnalité politique \\
\hline & $\begin{array}{l}\text { Appartient aux groupes : } \\
\text { Félibrige }\end{array}$ \\
\hline & Franc-Maçonnerie \\
\hline \multirow{3}{*}{$\begin{array}{l}\text { Proche du nouveau maire de Bordeaux, Adrien Marquet, il devient conservateur adjoint à la Bibliothèque municipale de } \\
\text { Bordeaux en } 1925 \text {; poste qu'il occupe jusqu'en } 1928 \text {, année où î quitte son emploi suite à une broulle avec Marquet pour se } \\
\text { lancer dans le joumalisme en tant que secrétaire de rédaction du joumal L'Avenir de la Vienne. }\end{array}$} & Institut d Estudis Occitans (I.E.O) \\
\hline & Parti Socialiste/SFO \\
\hline & Périodes \\
\hline \multirow{2}{*}{$\begin{array}{l}\text { Il devient directeur de ce journal en } 1929 \text { et le quitte en } 1932 \text { après ce qui semble être une longue succession de broulles qui le } \\
\text { voient notamment accusé d'être un sympathisant de l'Action Française. }\end{array}$} & 1876-1914 \\
\hline & $\begin{array}{l}1914-1939 \\
1939-1945\end{array}$ \\
\hline \multirow{2}{*}{$\begin{array}{l}\text { De retour en Gironde dans la maison familiale de Gaillan-en-Médoc, il tente de relancer sa carrière de journaliste en envoyant } \\
\text { des articles à divers joumaux et revues et essaie vainement de trouver une place au quotidien La Petite Gironde qui appartient } \\
\text { au même consortium que LAvenir de la Vienne. }\end{array}$} & $1945-1968$ \\
\hline & $\begin{array}{l}\text { Index géographique : } \\
\text { Bordeaux (Gironde) }\end{array}$ \\
\hline $\begin{array}{l}\text { Il fait aussi là ses premiers pas dans la politique en tant que candidat. Après avoir vainement tenté en } 1929 \text { d } \\
\text { E républicaine d'intérêts municipaux lors des élections municipales à poitters, il mène en tant que cal }\end{array}$ & $\begin{array}{l}\text { Paris (France) } \\
\text { Aquitaine (France) }\end{array}$ \\
\hline
\end{tabular}

Illustration 2.

\section{Problèmes et questionnements}

La mise en place du projet Vidas s'est rapidement heurté à quelques obstacles et a suscité chez ses fondateurs un certain nombre de questionnements.

Un premier écueil s'est rapidement fait jour après un premier appel à contributions lancé en 2012 et qui n'a reçu que peu de réponses, ces dernières se révélant par ailleurs souvent très vagues. De fait, la longueur de l'appel à contribution et surtout le fait que les fiches-exemples qui y été liées soient très complètes semble avoir rebuté les collaborateurs éventuels qui ont d'évidence craint de se lancer dans une entreprise trop contraignante pour eux.

La mise en ligne de première fiches sur le site Vidas, dont cer- taines «minimales», c'est-à-dire peu développées encore et se contentant d'informations basiques (dates et lieux de naissance et de morts lorsque l'on en dispose, quelques éléments biographiques succincts et quelques lignes sur les engagements dans la renaissance d'oc) a permis de passer ce premier obstacle en convaincant un certain nombre de collaborateurs putatifs de la possibilité de fournir des fiches sans avoir à se lancer dans d'interminables recherches.

Ainsi en va-t-il par exemple de la fiche consacrée à Maxence Bernheim de Villers (cf. illustration 3), acteur, certes, de second plan de la renaissance d'oc mais acteur tout de même et sur lequel, à l'occasion de leurs lectures ou de leurs dépouillements de documents, les chercheurs en 
les rédacteurs de réduire à un format de fiche des informations qui pourraient aisément (ou qui l'on déjà fait) aboutir à la rédaction de livres biographiques. C'est le cas, bien entendu d'hommes comme Frédéric Mistral, fondateur du Félibrige, ou Robert Lafont, acteur essentiel et central de l'occitanisme d'après-guerre.

Parce que, aussi, ces figures emblématiques, parfois idéalisées, parfois diabolisées ou écornées, prêtent bien entendu à la polémique. II convient dès lors de marcher sur des œufs, de savoir rester neutre, d'éviter les raccourcis sans pour autant trop entrer dans le détail. Autant de difficultés qui font que ces personnages qui doivent trouver leur place dans Vidas n'ont pas encore de fiche à leur nom, même si l'on ne désespère pas de pouvoir combler ces manques à moyen terme. II n'en demeure pas moins que cela participe aussi d'un questionnement plus large sur les priorités à accorder : fautil commencer par mettre en avant des personnages très connus et sur lesquels de nombreuses informations sont déjà disponibles, ou plutôt donner en premier lieu la priorité à des personnalités bien moins connues, pour lesquelles les informations sont plus difficilement accessibles, mais qui ont aussi joué un rôle important dans la renaissance d'oc ? Incontestablement, c'est plutôt vers cette seconde voie que le comité scientifique a choisi de s'engager pour commencer.

Tout cela explique le peu de fiches encore en ligne à ce jour ${ }^{4}$, mais il semble toutefois que le projet commence à trouver une certaine dynamique et quelque écho dans le milieu de la recherche en domaine occitan. Les propositions de fiches se font plus régulières au fur et à mesure que le site se rempli et les propositions de participation au projet se multiplient, laissant augurer d'un développement un peu plus conséquent dans les mois à venir.

Enfin, on peut espérer que ce travail de collaboration entre I'Université et le CIRDÒC, à travers notamment pour ce dernier l'administration technique du projet mais aussi une expertise essentielle dans le domaine archivistique, ouvre la voie à d'autres chantiers collectifs et vienne redynamiser la recherche en domaine occitan en lui offrant de nouveaux outils et champs de recherche.

Yan Lespoux, certifié d'occitan, docteur en histoire contemporaine (thèse soutenue en 2009 : Un demi-siècle de revendication en faveur de l'enseignement de la langue d'Oc (1940-1990) : enjeux, projets et réalisations), chercheur rattaché au laboratoire LLACS (Montpellier 3), membre du comité scientifique du projet Vidas.

4. Près de 30 à la fin de l'année 2015. 